\title{
To eat or not to eat: Cryo-EM structure of melanocortin receptor 4 reveals mechanism of a "hunger switch" initiating satiety signaling
}

\author{
Oksana Degtjarik ${ }^{1}$, Hadar Israeli ${ }^{1,2}$, Li F. Chan ${ }^{3}$, Danny Ben-Zvi' ${ }^{2}$ Masha Y. Niv ${ }^{4}$, Peter J. McCormick ${ }^{3}$, \\ Moran Shalev-Benami ${ }^{1}$
}

\author{
${ }^{1}$ Department of Chemical and Structural Biology, Weizmann Institute of Science, Rehovot, Israel; \\ ${ }^{2}$ Department of Developmental Biology and Cancer Research, The Hebrew University-Hadassah Medical School, Jerusalem, Israel; \\ ${ }^{3}$ Centre for Endocrinology, William Harvey Research Institute, Barts and the London School of Medicine, Queen Mary, University of \\ London, Charterhouse Square, London, UK; \\ ${ }^{4}$ Faculty of Agriculture, The Hebrew University, Israel;

\section{degtjarik648@gmail.com}

Obesity is a global epidemic causing increased morbidity and impaired quality of life. The melanocortin receptor 4 (MC4R) is a G protein-coupled receptor that plays a key role in regulation of food consumption and energy expenditure in the central nervous system, thus becoming a prime target for anti-obesity drugs. We present the cryo-EM structure of the human $\mathrm{MC}_{\mathrm{R}} \mathrm{R}-\mathrm{G}_{\mathrm{s}}$ signaling complex bound to the agonist setmelanotide, a cyclic peptide recently approved for the treatment of obesity. The work reveals the mechanism of MC4R activation, highlighting a molecular switch that initiates satiation signaling. Coupled to signaling assays and molecular dynamics simulations, the structure demonstrates the role calcium plays in receptor activation, but not inhibition. Altogether, these results fill a gap in understanding MC4R activation and provide guidelines for a structure-based design of novel and more efficient weight management drugs.

Keywords: GPCR, melanocortin, obesity, cryo-EM 